\title{
LUXAÇÃO RECIDIVANTE DO OMBRO: ASPECTOS DO PERIOODO ENTRE O PRIMEIRO EPISÓDIO E O TRATAMENTO CIRÚRGICO
}

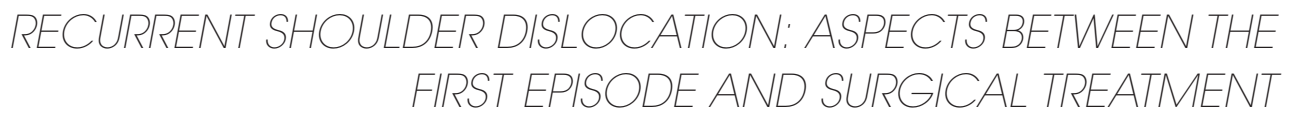

Roberto Yukio Ikemoto', Joel Murachovsky², Eric Strose ${ }^{3}$, Luís Gustavo Prata Nascimento ${ }^{3}$, Rogério Serpone Bueno ${ }^{3}$, Luís Henrique Oliveira Almeida ${ }^{3}$

\section{RESUMO}

Objetivo: Determinar: 1) se os pacientes que sofrem seu primeiro episódio de luxação traumática do ombro são orientados a manter imobilização por um período mínimo de quatro semanas e qual foi o tipo de imobilização prescrita; 2) determinar após quantos episódios de luxação os pacientes receberam a informação da necessidade de cirurgia; 3) quanto tempo os pacientes demoraram a conseguir acompanhamento com especialista em cirurgia de ombro; 4) quantos episódios de luxação os pacientes apresentavam no momento da cirurgia. Métodos: Dos 100 pacientes tratados cirurgicamente ou estão aguardando cirurgia nos ambulatórios, foram avaliados 61 , que responderam a um questionário com perguntas relacionadas com o mecanismo das luxações, locais de atendimento, orientações recebidas para o tratamento do evento agudo e acompanhamento, tempo necessário para conseguir o acompanhamento e cirurgia._Os dados coletados foram submetidos à análise. Resultados: Apenas 13 pacientes $(22 \%)$ receberam orientações adequadas sobre sua afecção, prognóstico quanto à recidiva e à necessidade de acompanhamento especializado e cirurgia nos casos recidivantes. Nenhum paciente foi orientado adequadamente como e por quanto tempo deveria ficar imobilizado. Conclusão: Nenhum paciente recebeu orientações de manter-se imobilizado por quatro semanas e o tipo de imobilização variou entre uma tipoia simples e a imobilização comercial tipo Velpeau. A maioria dos pacientes avaliados (78\%) não recebeu orientações corretas sobre a necessidade de acompanhamento especializado e cirurgia após o segundo episódio de luxação. O tempo para avaliação do especialista demorou entre quatro e seis meses e possuíam entre um e cem episódios de luxação no momento da cirurgia.

\section{ABSTRACT}

Objective: To determine: 1) whether the patients had been oriented to use immobilization for at least four weeks and which type of immobilization was prescribed, 2) how many dislocations occurred until the patient received information about the need of surgery, 3) How long it takes for patients to have an appointment with a shoulder surgeon, 4) How many dislocations the patient had at the time of surgery. Material and Methods: Of the 100 patients surgically treated or waiting for surgery at outpatient facilities, we interviewed 61 patients with questions related to the mechanism of dislocation, emergency service sites, guidelines for acute event treatment and follow-up, time elapsed until surgery and follow-up. Collected data were submitted to analysis. Results: Only 13 patients (22\%) had received correct information about their lesion, prognosis concerning recurrence, and about the need of surgery and expert follow-up in recurrent cases. None of our patients received proper information about type and duration of immobilization. Conclusion: None of our patients had received proper orientation to remain immobilized for four weeks, and the types of immobilization vary from a handmade sling to a manufactured Velpeau. Most of our patients (78\%) did not receive proper orientation about specialized follow-up and surgery after their second episode of dislocation. The time for a specialized appointment with shoulder surgeon ranges from four to six months, with 1-100 dislocation episodes at the moment of surgery.

Keywords - Shoulder joint; Shoulder dislocation; Epidemiology
Descritores - Articulação do ombro; Luxação do ombro; Epidemiologia
1 - Mestre em Medicina; Chefe do Grupo de Ombro e Cotovelo da Disciplina de Doenças do Aparelho Locomotor da FMABC.
2 - Doutor em Medicina; Assistente do Grupo de Ombro e Cotovelo da Disciplina de Doenças do Aparelho Locomotor da FMABC.
3 - Assistente do Grupo de Ombro e Cotovelo da Disciplina de Doenças do Aparelho Locomotor da FMABC. Correspondência: Eric Strose. Rua Joaquim Távora, 321/22, Vila Assunção, Santo André, SP. E-mail: ericstrose@hotmail.com 


\section{INTRODUÇÃO}

Em nosso meio, temos um grande déficit assistencial na saúde. Os pacientes sem um plano de saúde têm grande dificuldade para encontrar serviços públicos que possuam subespecialistas que tratem das mais diversas afecções clínicas ou cirúrgicas, dentre elas, as ortopédicas.

Muitos pacientes com luxação recidivante anterior traumática do ombro, que poderiam ser tratados cirurgicamente em um período precoce, acabam demorando em conseguir acompanhamento e cirurgia por falta de orientação adequada no momento do seu primeiro episódio.

$\mathrm{Na}$ literatura, encontramos trabalhos relatando pior prognóstico do resultado do tratamento cirúrgico quando os pacientes são tratados após vários episódios de luxação. Quanto maior o número de luxações, maior o grau de dificuldade de seu tratamento devido a desinserções labioglenoidais e capsuloligamentares - lesão de Bankart e a outras estruturas do ombro ${ }^{(1-3)}$.

Boileau et $a l^{(4)}$ demonstraram que a distensão dos ligamentos glenoumerais inferiores, como consequência da deformidade plástica sofrida por repetidos episódios de luxação e/ou subluxação, é fator de recidiva após a cirurgia para correção da instabilidade.

Como complicações da luxação recidivante do ombro, Buscayret et al ${ }^{(5)}$ encontraram relação estatisticamente significante entre o número de episódios e o desenvolvimento de artrose do ombro.

O objetivo do nosso trabalho foi determinar: 1) se os pacientes que sofrem seu primeiro episódio de luxação traumática do ombro são orientados a manter imobilização por um período mínimo de quatro semanas e qual foi o tipo de imobilização prescrita; 2) determinar após quantos episódios de luxação os pacientes receberam a informação de necessidade de cirurgia; 3 ) quanto tempo os pacientes demoram a conseguir um acompanhamento com especialista em cirurgia de ombro; 4) quantos episódios de luxação os pacientes apresentavam no momento da cirurgia.

\section{MÉTODOS}

De maio a julho de 2007, atendemos 61 pacientes em nosso ambulatório com luxação recidivante traumática do ombro. Quarenta e quatro pacientes $(72,14 \%)$ já haviam sido operados e 17 (27,86\%) aguardavam cirurgia. Do total dos pacientes, seis eram do gênero feminino $(9,84 \%)$ e 55 do masculino $(90,16 \%)$, com idade que variava de 18 a 59 anos (média de 31 anos e três meses).

A média de idade dos pacientes do sexo feminino foi de 32 anos e quatro meses (entre 22 e 41 anos) e do masculino, de 31 anos e dois meses (entre 18 e 59 anos).
A média do seguimento dos pacientes operados foi de 11,7 meses (entre um e 36 meses).

Dentre os 61 pacientes, a média de idade no momento do primeiro episódio de luxação era de 24 anos (entre 11 e 39 anos).

Os pacientes foram entrevistados e responderam a um questionário padrão por nós elaborado, que continha perguntas relacionadas com o mecanismo da primeira luxação, orientações recebidas para o tratamento do evento agudo e acompanhamento, tempo necessário para conseguir o acompanhamento e cirurgia, momento em que recebeu orientações adequadas e encaminhamento para cirurgia e número de episódios de luxação e/ou subluxação no momento da cirurgia, nos casos operados (Quadro 1).

Quadro 1 - Questionário utilizado para avaliação

\begin{tabular}{|l|l|l|l|l|}
\hline & Sim & Não & $\begin{array}{c}\text { Tempo / } \\
\text { imobilização }\end{array}$ & Episódios \\
\hline $\begin{array}{l}\text { Existiu trauma em seu primeiro episódio } \\
\text { de luxação? }\end{array}$ & & & & \\
\hline $\begin{array}{l}\text { Você recebeu orientação sobre como } \\
\text { deveria tratar seu 10 episódio de luxação? }\end{array}$ & & & & \\
\hline $\begin{array}{l}\text { Você permaneceu quatro semanas } \\
\text { imobilizado após 0 1 episódio de } \\
\text { luxação? Qual tipo de imobilização? }\end{array}$ & & & & \\
\hline $\begin{array}{l}\text { Você foi orientado em seu 1 }{ }^{\circ} \text { episódio de } \\
\text { luxação da necessidade de cirurgia caso } \\
\text { ocorressem novos episódios? }\end{array}$ & & & & \\
\hline $\begin{array}{l}\text { Você recebeu encaminhamento para } \\
\text { acompanhamento especializado das suas } \\
\text { luxações? Após quantos episódios? }\end{array}$ & & & & \\
\hline $\begin{array}{l}\text { Quanto tempo você levou para conseguir } \\
\text { seguimento especializado? }\end{array}$ & & & & \\
\hline $\begin{array}{l}\text { Quantos episódios de luxação no } \\
\text { momento da cirurgia? }\end{array}$ & & & & \\
\hline
\end{tabular}

Fonte: Ambulatório de Cirurgia de Ombro e Cotovelo - Hospital Mário Covas - FMABC

\section{RESULTADOS}

No atendimento do primeiro e segundo episódios de luxação, apenas 13 pacientes $(22 \%)$ receberam orientações adequadas sobre sua lesão, prognóstico quanto à recidiva, necessidade de cirurgia e necessidade de acompanhamento especializado, demorando em média quatro meses para obter acompanhamento (entre um e 14 meses). Desses pacientes, nove já foram submetidos à cirurgia e demoraram, em média, cinco meses para conseguir acompanhamento; no momento da cirurgia possuíam em média 10 episódios de luxação (entre um e 30 episódios).

Quando as orientações foram recebidas entre o terceiro e o $10^{\circ}$ episódio de luxação, o que ocorreu em 24 pacientes (39\%), o intervalo de tempo para obtenção do acompanhamento foi de seis meses (entre um e 60 meses); o número 
de episódios no momento da cirurgia, dentre os pacientes operados (16 pacientes), foi, em média, de 11 (entre quatro e 30$)$. Os 24 pacientes restantes (39\%) receberam as orientações após o $10^{\circ}$ episódio de luxação, conseguindo acompanhamento após cinco meses e meio, em média (entre um e 60 meses); possuíam, em média, no momento da cirurgia, quando operados (19 pacientes), 29 episódios de luxação (entre 11 e 100 episódios) (Tabela 1). Considerando todo o grupo, os pacientes receberam orientações adequadas sobre sua lesão e a necessidade de cirurgia no seu $13^{\circ}$ episódio de luxação e, quando submetidos à cirurgia, apresentavam 18 episódios de luxação, em média.

A variação no número de episódios no momento da cirurgia e o tempo necessário para obtenção do acompanhamento médico especializado estão expostos nas Figuras 1, 2 e 3.

Nenhum paciente foi orientado a permanecer imobilizado por um período mínimo de quatro semanas após seu primeiro episódio de luxação traumática do ombro, mesmo os 13 que foram orientados adequadamente sobre sua lesão, prognóstico quanto à recidiva, necessidade de cirurgia e necessidade de acompanhamento especializado.

\section{DISCUSSÃO}

Diversos fatores influenciam a recorrência da luxação anterior traumática do ombro após o seu primeiro episódio. Dentre eles podemos citar: idade do paciente no momento do primeiro episódio, sexo, participação em esportes de

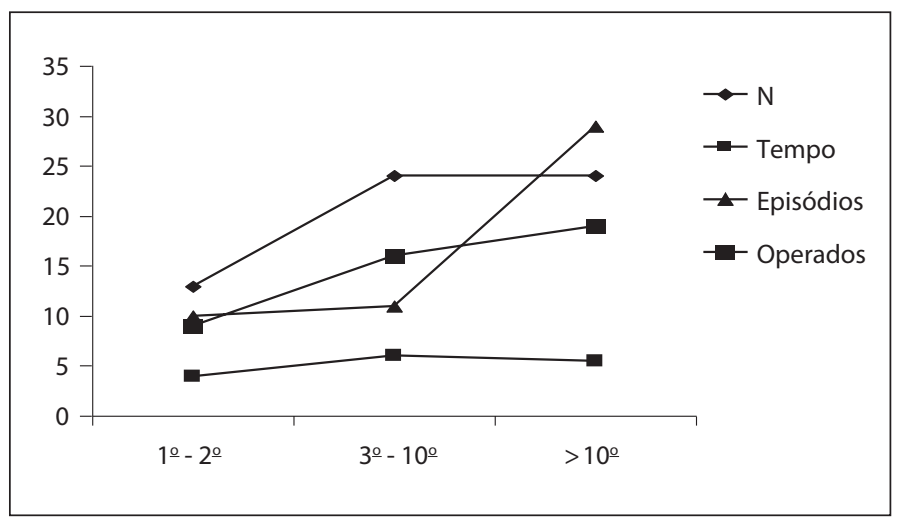

Fonte: Amb. Ombro e Cotovelo FMABC e HI

Figura 1 - Variação do número de episódios no momento da cirurgia

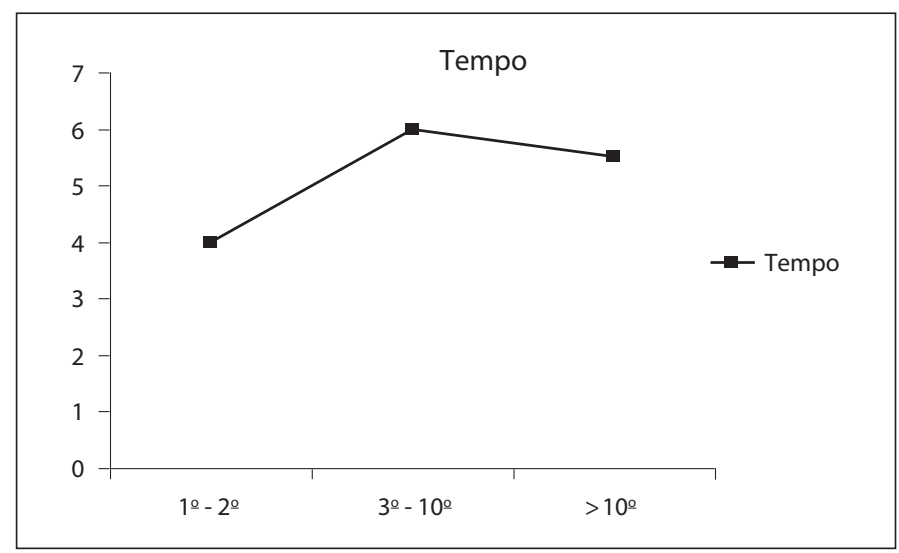

Fonte: Amb. Ombro e Cotovelo FMABC e HI

Figura 2 - Intervalo de tempo (em meses) para obtenção do acompanhamento médico especializado

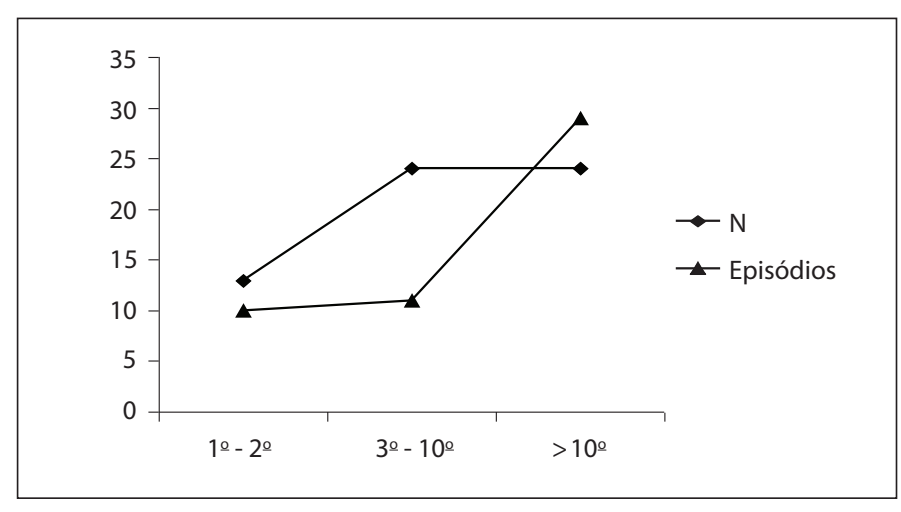

Fonte: Amb. Ombro e Cotovelo FMABC e HI

Figura 3 - Número de episódios no momento da cirurgia

contato, acometimento do membro dominante, tempo e tipo de imobilização utilizada, qualidade do complexo capsuloligamentar, presença da lesão de Bankart, presença de lesão de Hill-Sachs, presença de erosão da borda anteroinferior da cavidade glenoidal, entre outros ${ }^{(6-12)}$.

Alguns autores relatam que o fator mais determinante para a recidiva seja a idade do paciente no momento do primeiro episódio de luxação, estando os menores de 18 anos mais suscetíveis a apresentar recidiva da luxação, chegando a índices de até $86,7 \%(6,7,10,11)$.

Gartsman et $a l^{(3)}$ observaram aumento na gravidade das lesões labiais durante a artroscopia em pacientes com mais de três episódios de luxação; contudo, isso não influiu de maneira negativa nos resultados da cirurgia,

Tabela 1 - Avaliação do questionário

\begin{tabular}{|c|c|c|c|c|c|}
\hline Episódios & $\begin{array}{c}\text { Orientações para quatro } \\
\text { semanas tempo de imobilização }\end{array}$ & $\begin{array}{l}\text { Orientações corretas sobre } \\
\text { necessidade de cirurgia }\end{array}$ & $\begin{array}{c}\text { Tempo até avaliação } \\
\text { especializada }\end{array}$ & $\begin{array}{l}\text { №. luxações até a } \\
\text { cirurgia }\end{array}$ & $\mathrm{N}^{0}$. operados \\
\hline $1^{0}-2^{0}$ episódio & $0 \%$ & $13(22 \%)$ & $4 m(1-14)$ & $10(1-30)$ & 9 \\
\hline $3^{\circ}-10^{\circ}$ episódio & $0 \%$ & $24(39 \%)$ & $6 m(1-60)$ & $11(4-30)$ & 16 \\
\hline Após $10^{\circ}$ episódio & $0 \%$ & $24(39 \%)$ & $5,5 m(1-60)$ & $29(11-100)$ & 19 \\
\hline
\end{tabular}


inclusive nos índices de recidiva pós-operatória. Boileau et $a l^{(4)}$, em seu trabalho de 2006, observaram que os fatores que predispõem à recidiva pós-operatória são perda óssea substancial, seja no úmero ou na cavidade glenoidal, e distensão capsular provocada por repetidos episódios de luxação (Figuras 4 e 5). Outros autores também têm demonstrado que a erosão na borda anteroinferior da cavidade glenoidal é um fator importante no aumento da taxa de recidiva dos pacientes operados por luxação recidivante traumática anterior do ombro ${ }^{(4,13-16)}$.

Em nosso estudo, os pacientes apresentavam em média 18 episódios de luxação no momento da cirurgia, que julgamos ser um número elevado e injustificável de episódios para uma afecção que tem sua indicação cirúrgica classicamente orientada após o seu segundo episódio. Quando excluímos os 13 pacientes que foram orientados

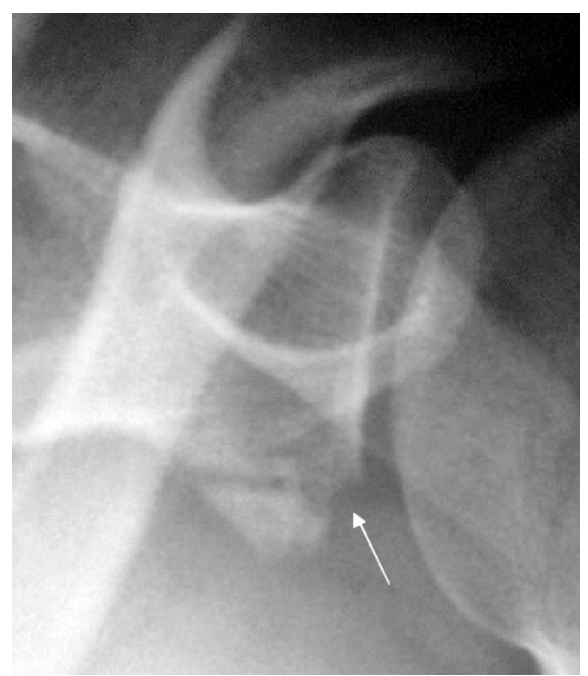

Figura 4 - Radiografia com fratura da borda da glenoide em paciente com dez episódios de luxação

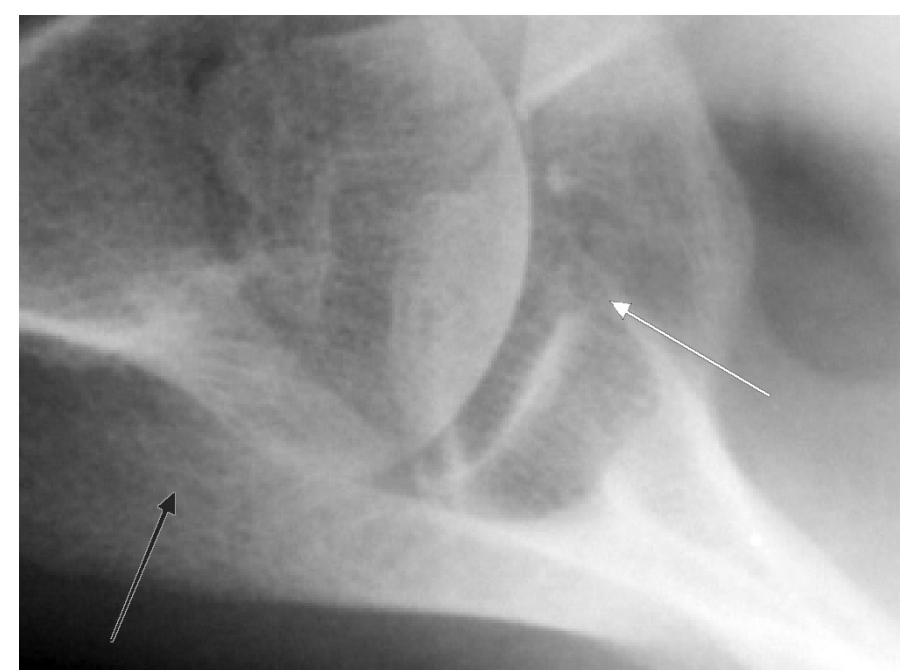

Figura 5 - Lesão de Hill-Sachs (seta preta) e erosão anterior da glenoide (seta branca) em paciente com 50 episódios de luxação adequadamente no $1^{\circ}$ e $2^{\circ}$ episódios de luxação, encontramos média de 21 episódios de luxação no momento da cirurgia dentre os restantes 35 pacientes operados; isso porque, em média, esses pacientes foram orientados apenas no $17^{\circ}$ episódio de luxação. Assim como Gartsman et $a l^{(3)}$, acreditamos que um número maior de episódios produz maiores lesões capsulares e labiais, o que dificulta a reparação durante a cirurgia; porém, discordamos do fato de o número de episódios não influir negativamente nos resultados pós-operatórios, principalmente nas recidivas, talvez por nossos pacientes apresentarem maior número de episódios de luxação no momento da cirurgia.

Além disso, esse número excessivo de episódios pode levar à erosão a borda anteroinferior da cavidade glenoidal, tornando o tratamento mais difícil; em alguns casos se torna necessário o uso de enxerto ósseo para restaurar a falha na cavidade glenoidal.

Burkhart e De Beer ${ }^{(13)}$ definiram que o formato da cavidade glenoidal, semelhante ao de uma "pera invertida" durante o procedimento artroscópico, indicaria perda óssea substancial da borda anterior e inferior da cavidade glenoidal, recomendando nesses casos o reparo da lesão com enxerto ósseo. Em média, a distância do ponto central da cavidade glenoidal até sua borda anterior seria de $11 \mathrm{~mm}$. Utilizamos esses parâmetros de avaliação artroscópica, associados à mensuração direta da perda óssea da borda anteroinferior da glenoide com probe milimetrado para definir a necessidade do uso de enxerto ósseo nos pacientes submetidos à cirurgia em nosso serviço.

Outras complicações podem ser esperadas em função da instabilidade do ombro. Samilson e Prieto ${ }^{(17)}$ descreveram que a instabilidade do ombro poderia ser causa de artrose em pacientes submetidos ou não a cirurgia. Denominaram tal afecção de dislocation arthropathy e desenvolveram uma classificação para a mesma. Em seu estudo, havia relação significativa entre a idade avançada no momento do primeiro episódio e o desenvolvimento de artrose do ombro. Matsoukis et al ${ }^{(18)}$ publicaram seus resultados de artroplastia do ombro em pacientes com artrose secundária à instabilidade do ombro, com relativos altos índices de complicações e reoperações. Importante observar nesse trabalho a rápida progressão do quadro degenerativo induzido pelos inúmeros episódios de luxação, tornando algumas vezes necessária uma artroplastia em pacientes com menos de 30 anos de idade.

Tendo em vista que nossos pacientes apresentam em média 18 episódios de luxação no momento de sua cirurgia, acreditamos que, assim como Buscayret et al $l^{(5)}$, boa parte desses pacientes que estamos acompanhando em nosso serviço poderá evoluir para artropatia dege- 
nerativa precoce por instabilidade do ombro e, portanto, necessitará uma artroplastia (Figuras 6 e 7).

Somando-se a isso, observamos que nenhum dos nossos pacientes foi bem orientado quanto ao período e tipo de imobilização necessário após o primeiro episódio de luxação.

Em nossa opinião, alguns motivos levam esses pacientes a não serem adequadamente orientados a tratar de maneira pertinente seu $1^{\circ}$ episódio de luxação traumática anterior do ombro. Dentre eles podemos citar o não conhecimento do tratamento apropriado pelo profissional que atendeu esse paciente, a falta de compreensão do tratamento orientado pelo profissional que o assistiu e a preocupação deste último em tratar exclusivamente o episódio de luxação do ombro e não as possíveis lesões já existentes.

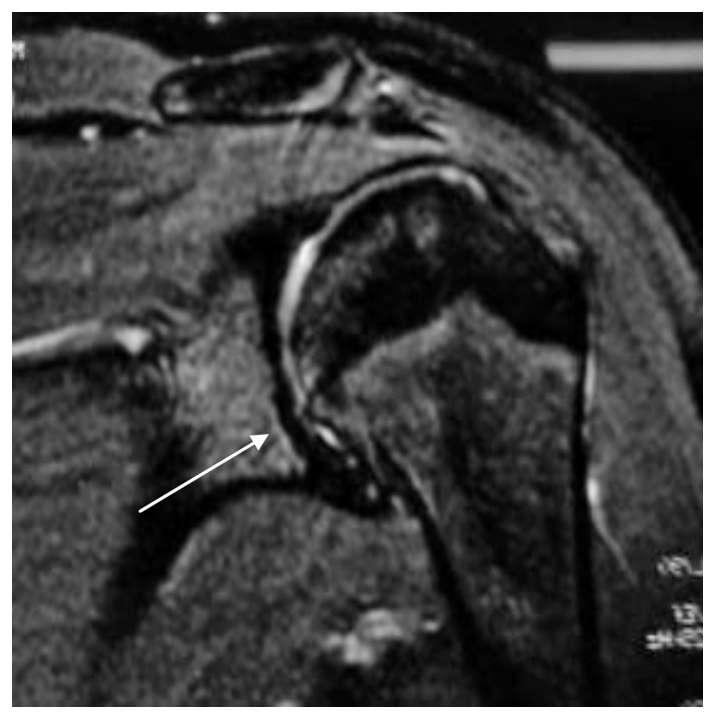

Figura 6 - Imagem de RNM mostrando pinçamento articular e osteófito marginal em paciente com 30 episódios de luxação

\section{REFERÊNCIAS}

1. Bankart AS, Cantab MC. Recurrent or habitual dislocation of the shoulder-joint. Br Med J. 1923;2(3285):1132-3.

2. Bankart AS. The pathology and treatment of recurrent dislocation of the shoulder-joint. Br J Surg. 1938;26(1):23-9.

3. Gartsman G, Roddey TS, Hammerman S. Arthroscopic treatment of anterior-inferior glenohumeral instability: two to five-year follow-up. J Bone Joint Surg. 2000;82(7):991-1003.

4. Boileau P,Villalba M, Héry JY, Balg F, Ahrens P, Neyton L. Risk factors for recurrence of shoulder instability after arthroscopic Bankart repair. J Bone Joint Surg Am. 2006;88(8):1755-63.

5. Buscayret F, Edwards TB, Szabo I, Adeleine P, Caudane H, Walch G. Glenohumera arthosis in anterior instability before and after surgical treatment. Am J Sports Med. 2004;32(5):1165-72.

6. Hovelius L, Lind B, Thorling J. Primary dislocation of the shoulder: factors affecting the two year prognosis. Clin Orthop Relat Res. 1983;(176):181-5.

7. Robinson CM, Howes J, Murdoch H, Will E, Graham C. Functional outcome and risk of recurrent instability after primary traumatic anterior shoulder dislocation in young patients. J Bone Joint Surg. 2006;88(11):2326-36.

8. Itoi E, Sashi R, Minagawa H, Shimizu T, Wakabayashi I, Sato K.. Position of immobilization after dislocation of the glenohumeral joint. J Bone Joint Surg Am. 2001;83(7):661-7.

9. Sugaya H, Moriishi J, Dohi M, Kon Y, Tsuchiya A. Glenoid rim morphology in recurrent anterior glenohumeral instability. J Bone Joint Surg Am. 2003;85(5):878-84.

10. te Slaa RL, Brand R, Marti RK. A prospective arthroscopic study of acute first-time

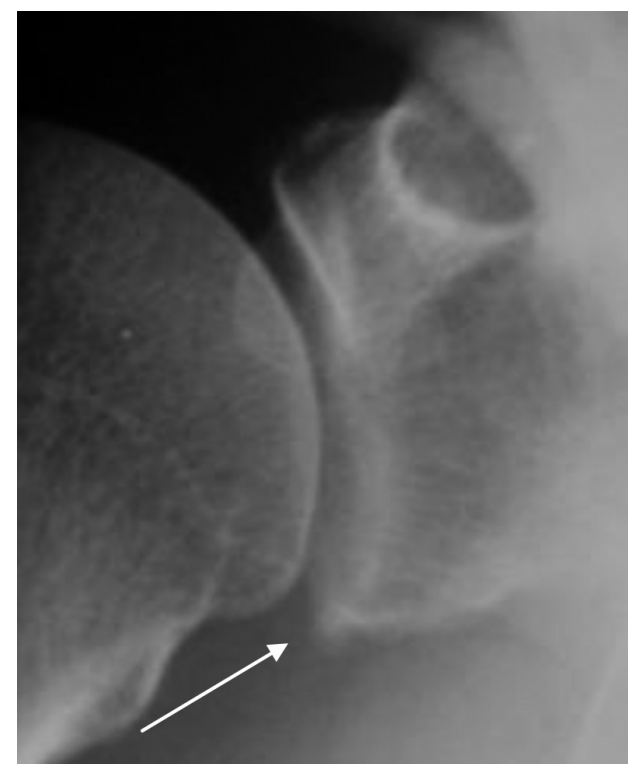

Figura 7 - Radiografia simples do ombro mostrando osteófito na glenoide em paciente com 20 episódios de luxação

\section{CONCLUSÃO}

1) Nenhum paciente recebeu orientações de manterse imobilizado por quatro semanas e o tipo de imobilização variou entre uma tipoia simples e a imobilização comercial tipo Velpeau.

2) A maioria dos pacientes avaliados (78\%) não recebeu orientações corretas sobre a necessidade de acompanhamento especializado e cirurgia após o segundo episódio de luxação.

3) O tempo para avaliação do especialista demorou entre quatro e seis meses.

4) Os pacientes possuíam entre um e 100 episódios de luxação no momento da cirurgia.

anterior shoulder dislocation in the young: a five-year follow-up study. J Shoulder Elbow Surg. 2003;12(6)529-34.

11. te Slaa RL, Wijffels MP, Brand R, Marti RK. The prognosis following acute primary glenohumeral dislocation. J Bone Joint Surg Br. 2004;86(1):58-64.

12. Robinson CM, Dobson RJ. Anterior instability of the shoulder after trauma. J Bone Joint Surg Br. 2004;86(4):469-79.

13. Burkhart SS, De Beer JF. Traumatic glenohumeral bone defects and their relationship to failure of arthroscopic Bankart repairs: significance of the inverted-pear glenoid and the humeral engaging Hill-Sachs lesion. Arthroscopy. 2000;16(7):677-94.

14. Itoi E, Lee SB, Berglund LJ, Berge LL, An KN. The effect of a glenoid defect on anteroinferior instability of the shoulder after Bankart repair: a cadaveric study. $J$ Bone Joint Surg Am. 2000;82(1):35-46.

15. Calvo E, Granizo JJ, Fernández-Yruegas D. Criteria for arthroscopic treatment of anterior instability of the shoulder: a prospective study. J Shouder Elbow Surg. 2005;87(5):677-83

16. Tauber M, Resch H, Forstner R, Raffl M, Schauer J. Reasons for failure of the surgical repair of anterior shoulder instability. J Shoulder Elbow Surg. 2004;13(3):279-85.

17. Samilson RL, Prieto V. Dislocation arthropathy of the shoulder. J Bone Joint Surg Am. 1983;65(4):456-60.

18. Matsoukis J, Tabib W, Guiffault P, Mandelbaum A, Walch G, Némoz C, Edwards B. Shoulder arthroplasty in patients with a prior anterior shoulder dislocation: results of a multicenter study. J Bone Joint Surg. 2003;85(8):1417-24. 